\title{
Risk factors associated with bleeding after prophylactic endoscopic variceal ligation in cirrhosis
}

Authors

Andreas Drolz ${ }^{*}, 1$, Christoph Schramm ${ }^{*}, 2$, Oliver Seiz ${ }^{1}$, Stefan Groth ${ }^{3}$, Eik Vettorazzi ${ }^{4}$, Thomas Horvatits ${ }^{1}$, Malte H. Wehmeyer ${ }^{1}$, Christoph Schramm¹, Tobias Goeser², Thomas Roesch ${ }^{3}$, Ansgar W. Lohse ${ }^{1}$, Johannes Kluwe ${ }^{1}$

\section{Institutions}

1 Department of Internal Medicine I, University Medical Center Hamburg Eppendorf, Hamburg, Germany

2 Department of Gastroenterology and Hepatology, University Hospital Cologne, Cologne, Germany

3 Department of Interdisciplinary Endoscopy, University Medical Center Hamburg Eppendorf, Hamburg, Germany

4 Department of Medical Biometry and Epidemiology, University Medical Center, Hamburg, Germany

submitted 4.9.2019

accepted after revision 2.6.2020

published online 7.9 .2020

Bibliography

Endoscopy 2021; 53: 226-234

DOI 10.1055/a-1214-5355

ISSN 0013-726X

(C) 2020. Thieme. All rights reserved.

Georg Thieme Verlag KG, Rüdigerstraße 14,

70469 Stuttgart, Germany

\section{Corresponding author}

Johannes Kluwe, MD, Department of Internal Medicine I, University Medical Center Hamburg-Eppendorf, Martinistraße 52, Hamburg 20246, Germany

Fax: +49-40-74100

j.kluwe@uke.de

Supplementary material

Supplementary material is available under

https://doi.org/10.1055/a-1214-5355

Scan this QR-Code for the author commentary.

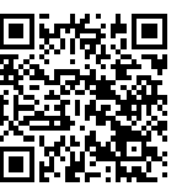

\footnotetext{
* These authors contributed equally to this work.
}

\section{GRAPHICAL ABSTRACT}

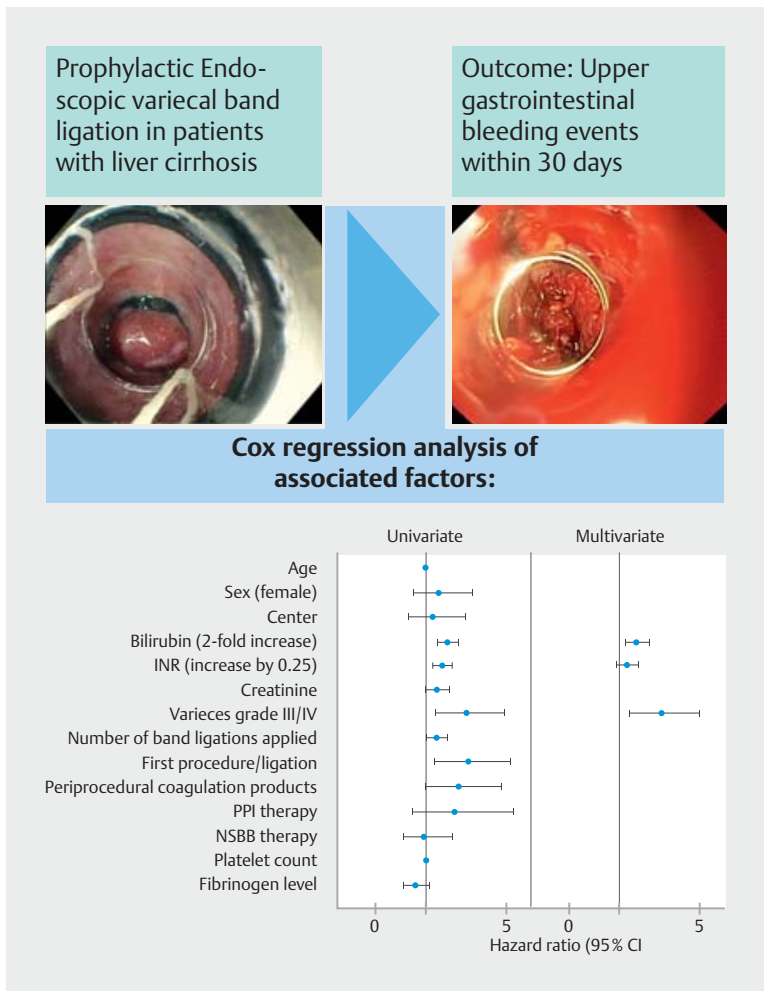

ABSTRACT

Background Prophylactic endoscopic variceal band ligation (EVL) is frequently performed in patients with liver cirrhosis. The aim of our study was to identify factors associated with early upper gastrointestinal bleeding (UGIB) in cirrhosis patients after prophylactic EVL.

Methods 787 nonemergency EVLs performed in 444 patients in two German University medical centers were analyzed retrospectively.

Results Within 30 days after EVL, 38 UGIBs were observed (4.8\% of all procedures). Bilirubin levels (hazard ratio [HR] 1.5, $95 \%$ confidence interval $[\mathrm{Cl}] 1.2-2.0$ for a 2 -fold increase) and presence of varices grade III/IV according to Paquet (HR 2.6, 95\% Cl 1.3-5.0 compared with absence or smaller sized varices) were independently associated with UGIB following EVL. International normalized ratio (INR) was associated with bleeding events in the univariate analysis but did not reach statistical significance after adjust- 
ment for bilirubin and presence of varices grade III/IV (HR $1.2,95 \% \mathrm{Cl} 0.9-1.6$ for an increase by 0.25 ). There was no statistically significant association between platelet count or fibrinogen levels and UGIB. Substitution of coagulation products did not affect incidence of bleeding after EVL, which also applied to patients with "coagulopathy" (INR > 1.5 and/or platelet count $<50 \times 10^{9} / \mathrm{L}$ ). No association be- tween proton pump inhibitor therapy and post-EVL UGIB was observed.

Conclusions EVL is a safe procedure and immediate bleeding complications are rare. Serum bilirubin levels and size of varices, rather than coagulation indices, are associated with UGIB after EVL. Our data do not support the preventive substitution of blood or coagulation products.

\section{Introduction}

Bleeding from esophageal or gastric varices is a life-threatening complication resulting from portal hypertension in liver cirrhosis $[1,2]$. Esophageal varices are present in $40 \%-60 \%$ of cirrhosis patients, and annual incidence of first variceal bleed is estimated to be $4 \%[3,4]$. Endoscopic variceal band ligation (EVL) with or without nonselective beta-blocker therapy is a central element of primary and secondary bleeding prophylaxis in patients with medium and large esophageal varices [5,6], and has been shown to be effective in reducing bleeding complications [7,8].

Yet, an important potential complication of EVL is procedure-related bleeding, which has been described in $2 \%-6 \%$ of cirrhosis patients undergoing EVL [9-11]. However, risk factors for bleeding complications in patients receiving EVL are not well established. Changes in routine coagulation test results are a frequent finding in patients with liver cirrhosis. The typical laboratory pattern of hepatic coagulopathy often includes reduced platelet counts (and function), elevated international normalized ratio (INR), and decreased fibrinogen levels [1214]. Whether these changes directly translate into an increased bleeding risk remains a matter of debate. At least under stable conditions, a rebalanced hemostasis has been suggested in patients with chronic liver disease, mainly attributable to a reduction of both pro- and anticoagulatory factors $[12,15]$.

The primary aim of this study was to determine factors associated with upper gastrointestinal bleeding (UGIB) in cirrhosis patients within 30 days after prophylactic EVL. The secondary aim was to assess the impact of substitution of blood/coagulation products on bleeding risk in these patients.

\section{Methods}

A total of 1006 prophylactic, nonemergency EVL procedures performed in an inpatient setting were retrospectively identified and studied at the University Medical Centers Hamburg and Cologne between 01/2009 and 12/2017. Only patients with liver cirrhosis were eligible for inclusion in the study. Patients with missing laboratory or follow-up data were excluded from analysis. Fig. 1 shows a detailed study flow chart.

All data were extracted from the prospectively maintained patient data management systems used by the participating centers. Only patients with laboratory analyses performed prior to EVL were included in the study. Platelet count, INR, serum bilirubin, and creatinine were defined as mandatory variables in the preinterventional assessment and were documented. If available, fibrinogen levels were also documented.

The cause of liver disease was documented in all patients, and severity of liver disease was assessed by the Model of EndStage Liver Disease (MELD) score [16]. Findings during endoscopy (size of varices, presence of red spots, number of ligations) were also evaluated.

Patients with available follow-up data for at least 30 days after EVL were included and procedure-related complications were assessed. Follow-up data were obtained directly from the patients at the following admission or at follow-up outpatient visits, respectively.

This noninterventional study was conducted in accordance with the Declaration of Helsinki and Good Clinical Practice guidelines, and was approved by the ethics committees of the Hamburg Medical Chamber (reference number WF-63/16) and the Medical Faculty of the University of Cologne (reference number 19-162). Informed consent was waived due to the retrospective and observational character of the study.

\section{Patient management}

Patients were managed based on the current Baveno recommendations [5]. Thus, only patients with medium to large varices underwent prophylactic EVL. Use of vasoactive agents, such as terlipressin, was restricted to patients with active bleeding, and was therefore not routinely used in the peri-interventional setting. Currently, no recommendations are available regarding management of hepatic coagulopathy [5]. Therefore, the deci-

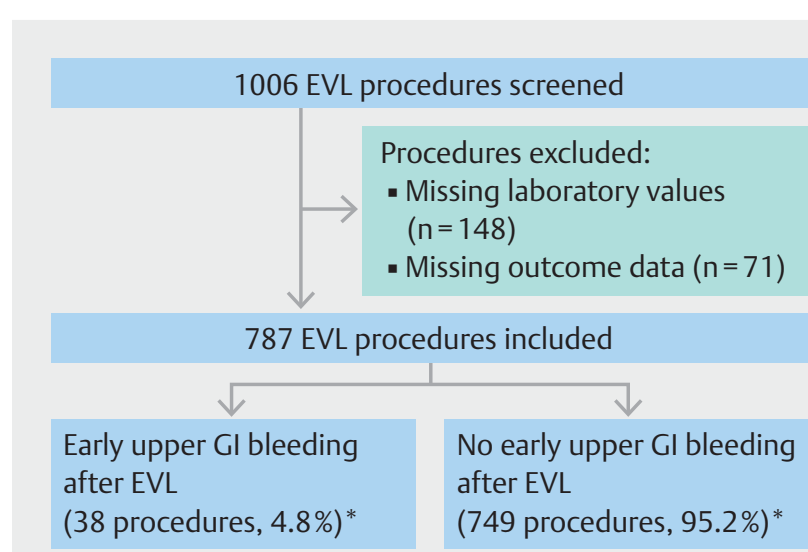

- Fig. 1 Study flow chart. EVL, endoscopic variceal band ligation; $\mathrm{GI}$, gastrointestinal. *Follow-up 30 days. 
sion for the preprocedural substitution of blood/coagulation products was based on the individual assessment by the attending physicians, as was the decision on the use of proton pump inhibitors (PPIs). If the decision was made to substitute blood/coagulation products, these were given shortly prior to the intervention on the same day. Following EVL, patients were instructed to stay on a diet of soft or liquid foods (e. g. mashed potatoes, cream soups) for a least 3 days.

\section{Definitions}

Prophylactic EVL was defined as nonemergency EVL for the purpose of prophylaxis of variceal bleeding in cirrhosis patients without active bleeding at the time of endoscopy [5, 6].

Presence of liver cirrhosis was defined by characteristic clinical (ascites, caput medusae, spider naevi, etc.), laboratory, and radiological findings (typical morphological changes of the liver, signs of portal hypertension, etc. in ultrasonography or computed tomography scanning), or via histology, if available [14].

Esophageal varices were graded according to the Paquet's classification [17].

Early UGIB following EVL was defined as presence of hematemesis, and/or melena, and/or firm clinical or laboratory evidence of acute blood loss from the upper gastrointestinal tract [18], which occurred within 30 days from EVL.

\section{Statistics}

Data are presented as median and interquartile range (IQR; $25 \%-75 \%$ percentiles), or number and percentage, respectively. Comparisons between groups were performed using chi-squared test, Fisher's exact test, or Mann-Whitney $U$ test, as appropriate. Spearman rank correlation was used to assess correlations between metric variables. A mixed Cox proportional hazards regression model with a normal frailty distribution was used to identify risk factors for bleeding after ligations. This model reflects the clustering structure induced by observing repeated events within the same patients [19, 20]. To avoid overfitting in the multivariate analysis, a stepwise variable selection procedure (with iterations between forward and backward steps) was applied. Additionally, the potential impact of coagulation product substitution on bleeding incidence was analyzed in a predefined coagulopathy subsample (patients with INR $>1.5$ and/or platelet count< $50 \times 10^{9} / \mathrm{L}$ ) using the aforementioned methods. As all the analyses were considered exploratory, no correction for multiple testing was performed and $P$ values of $<0.05$ were considered statistically significant.

Statistical analysis was conducted using IBM SPSS Statistics version 22 (IBM Corp., Armonk, New York, USA) and RStudio version 1.1.463 (R Foundation for Statistical Computing, Vienna, Austria).

\section{Results}

\section{Patients}

In total, 787 prophylactic EVL procedures performed in 444 patients were analyzed; 414 patients were undergoing their first EVL procedure. The median age at time of EVL was 59 years, and approximately $70 \%$ of the procedures were performed in male patients. Alcoholic and viral etiology accounted for more than $60 \%$ of patients/procedures. The median number of ligations applied during one session was 4 (IQR 3-5). Overall, 13 patients (2.9\% of patients; $1.7 \%$ of all EVL procedures) died during follow-up, with main causes of death being acute-onchronic liver failure and infections. Detailed patient characteristics are presented in $>$ Table 1. Typical endoscopic findings in patients with cirrhosis are shown in $\mathbf{F i g} \mathbf{2}$.

\section{Early UGIB after EVL}

During the 30 -day follow-up period, 38 UGIB events ( $4.8 \%$ of all EVLs) were observed at a median of 13 days (IQR 8-17) following EVL. Only three cases of procedure-related bleeding were observed within 24 hours after EVL (7.9\% of all UGIB events), all of which occurred during the procedure (one bleeding from the ligation ulcer; one bleeding due to accidental removal of the ligation band; one insufficient ligation resulting in bleeding). In 31 patients (81.6\%), ligation ulcers and/or esophageal varices were identified as the source of bleeding. One patient developed UGIB from gastric varices (2.6\%), and in six patients (15.8\%) the exact bleeding source could not be determined but was suspected to be in the upper gastrointestinal tract. Only two patients developed more than one bleeding event after EVL during the study period.

Most UGIBs were observed following the first EVL procedure $(73.7 \%$ of all observed post-procedural UGIBs). The rate of bleeding after EVL was $6.8 \%$ (95\% confidence interval $[\mathrm{Cl}]$ $4.5 \%-9.6 \%)$ after the first, $3.0 \%(95 \% \mathrm{Cl} 1.1 \%-6.4 \%)$ after the second, and $2.3 \%(95 \% \mathrm{Cl} 0.6 \%-5.9 \%)$ after the third or later EVL procedures. Table 1 shows detailed descriptive baseline characteristics of patients with and without bleeding within 30 days after EVL.

\section{Factors associated with early UGIB after EVL}

No significant difference in sex, age, or cirrhosis etiology was observed between patients with and without post-EVL UGIB. Bleeding rates were comparable between the two centers (University Medical Center Hamburg-Eppendorf 5.3\% vs. University Hospital Cologne $4.2 \%)$. There was no statistically significant association of peri-interventional use of PPI or nonselective beta-blockers with bleeding incidence following EVL ( $>$ Table 2; see also Fig. $1 \mathbf{s}$ in the online-only supplementary material). Periprocedural substitution of coagulation products was more frequently performed in patients who developed UGIB during follow-up ( $\triangleright$ Table 1); however, there was no significant association between substitution and occurrence of bleeding after EVL ( $\triangleright$ Table 2 ).

Of the routine coagulation tests assessed, only INR was significantly associated with UGIB in the univariate analysis ( $\triangleright \mathrm{Ta}$ ble 2); however, the absolute difference in INR between pa- 
- Table 1 Patient characteristics.

\begin{tabular}{|c|c|c|c|}
\hline Variable & $\begin{array}{l}\text { All EVL procedures } \\
(n=787)\end{array}$ & $\begin{array}{l}\text { Bleeding within } 30 \text { days } \\
(n=38)\end{array}$ & $\begin{array}{l}\text { No bleeding within } \\
30 \text { days } \\
(n=749)\end{array}$ \\
\hline Age, median (IQR), years & $59(50-66)$ & $55(49-64)$ & $59(50-66)$ \\
\hline Female sex, n (\%) & $237(30)$ & $14(37)$ & $223(30)$ \\
\hline Center (Hamburg / Cologne), n (\%) & $474(60) / 313(40)$ & $25(66) / 13(34)$ & $449(60) / 300(40)$ \\
\hline \multicolumn{4}{|l|}{ Cause of cirrhosis, n (\%) } \\
\hline - Alcohol & $274(35)$ & $15(39)$ & $259(35)$ \\
\hline - Viral & $176(22)$ & $12(32)$ & $164(22)$ \\
\hline - Mixed & $48(6)$ & $4(11)$ & $44(6)$ \\
\hline - Cryptogenic & $63(8)$ & $2(5)$ & $61(8)$ \\
\hline - Other & $226(29)$ & $5(13)$ & $221(29)$ \\
\hline MELD score, median (IQR) & $11(9-14)$ & $15(10-22)$ & $11(9-14)$ \\
\hline \multicolumn{4}{|l|}{ Band ligation } \\
\hline - Varices Paquet’s grade III/IV¹, n/N (\%) & $270 / 761(35)$ & $21 / 38(55)$ & $249 / 723(34)$ \\
\hline $\begin{array}{l}\text { - Risk factors (cherry red spots, large varices, thinning } \\
\text { of the variceal wall) })^{2}, \mathrm{n} / \mathrm{N}(\%)\end{array}$ & $581 / 697(83)$ & $34 / 37(92)$ & $547 / 660(83)$ \\
\hline - Number of band ligations applied ${ }^{3}$, median (IQR) & $4(3-5)$ & $5(4-6)$ & $4(3-5)$ \\
\hline $\begin{array}{l}\text { Peri-interventional substitution of coagulation products, } \\
n(\%)\end{array}$ & $116(15)$ & $10(26)$ & $106(14)$ \\
\hline PPI therapy ${ }^{4}, \mathrm{n} / \mathrm{N}(\%)$ & $613 / 771(80)$ & $32 / 36(89)$ & $581 / 735(79)$ \\
\hline NSBB therapy, n (\%) & $445(57)$ & $21(55)$ & $424(57)$ \\
\hline \multicolumn{4}{|l|}{ Laboratory tests, median (IQR) } \\
\hline . INR & $1.2(1.1-1.3)$ & $1.3(1.2-1.6)$ & $1.2(1.1-1.3)$ \\
\hline - Platelets, $10^{9} / \mathrm{L}$ & $92(63-130)$ & $92(65-137)$ & $92(63-130)$ \\
\hline - Fibrinogen, g/ $\mathrm{L}^{5}$ & $2.8(2.2-3.5)$ & $2.4(1.9-3.2)$ & $2.8(2.2-3.5)$ \\
\hline - Bilirubin, mg/dL & $1.5(0.9-2.7)$ & $2.8(1.4-8.9)$ & $1.4(0.9-2.6)$ \\
\hline - Creatinin, mg/dL & $0.9(0.7-1.1)$ & $1.0(0.8-1.2)$ & $0.9(0.71 .1)$ \\
\hline \multicolumn{4}{|l|}{ Complications within 30 days } \\
\hline - Death, n (\%) & $13(2)$ & $10(26)$ & $3(0.4)$ \\
\hline - GI Bleeding, n (\%) & $38(5)$ & $38(100)$ & 0 \\
\hline - Onset of bleeding, median (IQR), days after ligation & $13(8-17)$ & $13(8-17)$ & - \\
\hline \multicolumn{4}{|c|}{$\begin{array}{l}\text { EVL, endoscopic variceal band ligation; IQR, interquartile range; MELD, Model of End-Stage Liver Disease; PPI, proton pump inhibitor; NSBB, nonselective } \\
\text { beta-blocker; INR, international normalized ratio. } \\
{ }^{1} \text { Available in } 761 \text { cases }(97 \%) \text {. } \\
{ }^{2} \text { Available in } 697 \text { cases }(89 \%) . \\
{ }^{3} \text { Available in } 776 \text { cases }(99 \%) \\
{ }^{4} \text { Available in } 771 \text { cases }(98 \%) \\
5 \text { Available in } 559 \text { cases }(71 \%) .\end{array}$} \\
\hline
\end{tabular}

tients who did and did not develop bleeding was only marginal (1.3 [IQR 1.2-1.6] vs. 1.2 [IQR 1.1-1.3]). Moreover, the risk for UGIB was highest after the first EVL procedure ( $\triangleright$ Table 2 ). Other variables were also associated with EVL-related bleeding in the univariate analyses: presence of varices grade III/IV (com- pared with smaller sized varices or no varices at all), number of ligations applied, and serum bilirubin levels.

The multivariate mixed Cox proportional hazards model identified bilirubin and presence of varices grade III/IV as risk factors for bleeding ( $\vee$ Table 2 ). We found a considerable heterogeneity between patients even in the adjusted model in the 


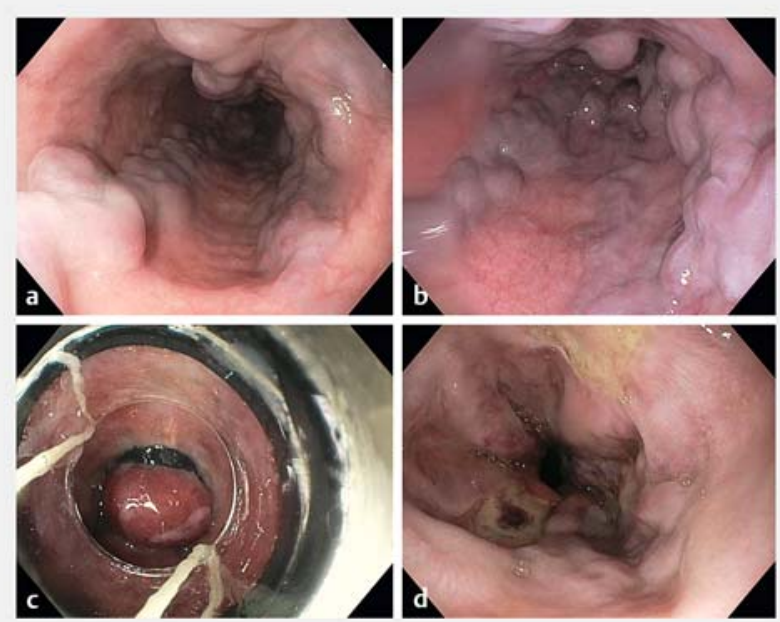

- Fig. 2 Typical endoscopic findings in a patient with cirrhosis. a,b Esophageal varices. c Variceal band ligation applied to varices. d Post-ligation ulcer as a potential source of bleeding. individual hazard of bleeding with an unexplained variance of the frailty distribution of 0.8 . This translates to a median hazard ratio (HR) of 2.4 [19], which is comparable to the effect found for varices grade III/IV.

\section{Coagulation product substitution}

In $116 \mathrm{EVL}$ procedures (14.7\%), patients received periprocedural coagulation product substitution, primarily platelets and/or fresh frozen plasma (FFP). In 52 of these cases (44.8\%), only platelets were substituted, 35 cases were managed using FFP only (30.2\%), 26 cases received FFP and platelets (22.4\%), and 3 cases were given coagulation factor concentrates (2.6\%). In cases of substitution, a median of 2 (IQR 2-2) FFPs and 1 (IQR 1-1) platelet concentrate were given. Table 3 presents the differences between EVL procedures with and without substitution in the total cohort.

\section{Effect of coagulation product substitution}

A "coagulopathy group" was defined by INR $>1.5$ and/or platelet count $<50 \times 10^{9} / \mathrm{L}$, which are frequently used as trigger values for coagulation product substitution in clinical practice. A

- Table 2 Estimated univariate and multivariate hazard ratios for upper gastrointestinal bleeding after endoscopic variceal band ligation from a mixed Cox proportional hazards model with a normally distributed frailty term.

\begin{tabular}{|c|c|c|}
\hline \multirow[t]{2}{*}{ Variable } & \multicolumn{2}{|c|}{ Hazard ratio $(95 \% \mathrm{Cl})$} \\
\hline & Univariate & Multivariate ${ }^{1}$ \\
\hline Age & $0.99(0.97-1.02)$ & \\
\hline Female sex & $1.38(0.68-2.79)$ & \\
\hline Center (Hamburg) & $1.19(0.58-2.44)$ & \\
\hline \multicolumn{3}{|l|}{ MELD score components } \\
\hline - Bilirubin (2-fold increase) & $1.68(1.34-2.12)^{3}$ & $1.54(1.18-2.02)^{3}$ \\
\hline - INR (0.25 increase) & $1.50(1.20-1.87)^{3}$ & $1.23(0.93-1.62)$ \\
\hline - Creatinine & $1.32(0.99-1.76)$ & \\
\hline \multicolumn{3}{|l|}{ Band ligation } \\
\hline - Varices Paquet's grade III/IV² & $2.48(1.28-4.84)^{3}$ & $2.58(1.32-5.04)^{3}$ \\
\hline - Number of band ligations applied ${ }^{4}$ & $1.31(1.02-1.68)^{3}$ & \\
\hline - First procedure/ligation & $2.57(1.23-5.35)^{3}$ & \\
\hline Peri-interventional substitution of coagulation products & $2.12(0.98-4.61)$ & \\
\hline PPI therapy ${ }^{5}$ & $1.95(0.67-5.67)$ & \\
\hline NSBB therapy & $0.94(0.48-1.86)$ & \\
\hline \multicolumn{3}{|l|}{ Other laboratory tests } \\
\hline - Platelets $\left(10^{9} / \mathrm{L}\right)$ & $1.00(0.99-1.00)$ & \\
\hline - Fibrinogen $(\mathrm{g} / \mathrm{L})^{4}$ & $0.72(0.47-1.10)$ & \\
\hline $\begin{array}{l}\mathrm{Cl} \text {, confidence interval; MELD, Model of End-Stage Liver Disease } \\
{ }^{1} \text { The multivariate model was fitted by a stepwise variable selec } \\
\left.{ }^{2} \text { Available in } 761 \text { cases ( } 97 \%\right) \text {. } \\
{ }^{3} \text { Indicates significant association. } \\
\left.{ }^{4} \text { Available in } 776 \text { cases ( } 99 \%\right) . \\
{ }^{5} \text { Available in } 771 \text { cases }(98 \%) .\end{array}$ & $\begin{array}{l}\text { ternational normalizec } \\
\text { cedure based on all va }\end{array}$ & $\begin{array}{l}\text { hibitor; NSBB, nonselective beta-blocker } \\
\text { ociations in the univariate analysis. }\end{array}$ \\
\hline
\end{tabular}


- Table 3 Endoscopic variceal band ligation procedures stratified according to peri-interventional substitution of blood/coagulation products.

\begin{tabular}{|c|c|c|c|c|c|c|}
\hline \multirow[t]{2}{*}{ Variable } & \multicolumn{3}{|c|}{ All EVLs $(n=787)$} & \multicolumn{3}{|c|}{ Coagulopathy group $(n=160)^{1}$} \\
\hline & $\begin{array}{l}\text { Substitution } \\
(n=116)\end{array}$ & $\begin{array}{l}\text { No substitution } \\
(\mathrm{n}=671)\end{array}$ & $P$ value & $\begin{array}{l}\text { Substitution } \\
(\mathrm{n}=98)\end{array}$ & $\begin{array}{l}\text { No substitution } \\
(n=62)\end{array}$ & $P$ value \\
\hline Age, median (IQR), years & $56(51-62)$ & $59(49-67)$ & 0.07 & $56(50-63)$ & $53(44-62)$ & 0.06 \\
\hline Female sex, n (\%) & $26(22)$ & $211(31)$ & 0.05 & $21(21)$ & $18(29)$ & 0.28 \\
\hline Center (Hamburg), n (\%) & $61(53)$ & $413(62)$ & 0.07 & $54(55)$ & $37(60)$ & 0.57 \\
\hline MELD score, median (IQR) & $15(11-19)$ & $11(8-14)$ & $<0.001$ & $15(11-19)$ & $14(11-19)$ & 0.39 \\
\hline \multicolumn{7}{|l|}{ Band ligation } \\
\hline $\begin{array}{l}\text { - Varices Paquet's grade III/IV², } \\
\text { n/N (\%) }\end{array}$ & $38 / 116(33)$ & $232 / 652(36)$ & 0.88 & 29/91 (32) & $20 / 60(33)$ & 0.85 \\
\hline $\begin{array}{l}\text { Risk factors (cherry red spots, } \\
\text { large varices, thinning of the } \\
\text { variceal wall) }{ }^{3}, \mathrm{n} / \mathrm{N}(\%)\end{array}$ & $78 / 98(80)$ & $503 / 599(84)$ & 0.28 & $63 / 82(77)$ & $52 / 58(90)$ & 0.05 \\
\hline $\begin{array}{l}\text { - Number of band ligations ap- } \\
\text { plied }^{4} \text {, median (IQR) }\end{array}$ & $4(3-6)$ & $4(3-5)$ & 0.19 & $4(3-6)$ & $4(3-5)$ & 0.06 \\
\hline \multicolumn{7}{|l|}{ Laboratory tests, median (IQR) } \\
\hline - INR & $1.4(1.3-1.6)$ & $1.2(1.1-1.3)$ & $<0.001$ & $1.4(1.3-1.6)$ & $1.4(1.2-1.6)$ & 0.60 \\
\hline - Platelets, $10^{9} / \mathrm{L}$ & $43(34-68)$ & $98(73-135)$ & $<0.001$ & $40(31-56)$ & $48(41-81)$ & $<0.01$ \\
\hline - Fibrinogen, $g / \mathrm{L}^{5}$ & $2.0(1.4-2.3)$ & $3.0(2.4-3.6)$ & $<0.001$ & $2.0(1.4-2.3)$ & $2.2(1.8-2.6)$ & 0.12 \\
\hline - Bilirubin, mg/dL & $2.5(1.3-4.8)$ & $1.4(0.9-2.3)$ & $<0.001$ & $2.3(1.3-4.6)$ & $2.2(1.0-4.7)$ & 0.46 \\
\hline - Creatinine, mg/dL & $0.9(0.7-1.2)$ & $0.9(0.7-1.1)$ & 0.13 & $1.0(0.7-1.2)$ & $0.9(0.7-1.1)$ & 0.29 \\
\hline \multicolumn{7}{|l|}{ Complications within 30 days, $\mathrm{n}(\%)$} \\
\hline - Bleeding & $10(9)$ & $28(4)$ & 0.04 & $7(7)$ & $5(8)$ & $>0.99$ \\
\hline - Death & $5(4)$ & $8(1)$ & 0.03 & $4(4)$ & $2(3)$ & $>0.99$ \\
\hline \multicolumn{7}{|c|}{$\begin{array}{l}\text { EVL, endoscopic variceal band ligation; IQR, interquartile range; MELD, Model of End-Stage Liver Disease; INR, international normalized ratio. } \\
{ }^{1} \text { Coagulopathy group defined by INR }>1.5 \text { and/or platelet count }<50 \times 10^{9} / \mathrm{L} \text {. } \\
{ }^{2} \text { Available in } 768(98 \%) / 151(94 \%) \text { cases. } \\
{ }^{3} \text { Available in } 697(89 \%) / 140(88 \%) \text { cases. } \\
{ }^{4} \text { Available in } 776(99 \%) / 158(99 \%) \text { cases. } \\
{ }^{5} \text { Available in } 559(71 \%) / 119(74 \%) \text { cases. }\end{array}$} \\
\hline
\end{tabular}

total of 160 procedures fulfilled these criteria. Median MELD score in this group was 14 (IQR 11-19). In total, 12 cases of UGIB after EVL (7.5\% of cases) were observed in this sample, and 6 patients (3.8\% of cases) died during follow-up. In this group, periprocedural coagulation products were given in 98 cases $(61.3 \%)$. There was a trend for higher bleeding rates in the "coagulopathy group" compared with patients without coagulopathy ( $7.5 \%$ vs. $4.1 \%)$; this association, however, failed to reach statistical significance $(P=0.08)$. Characteristics of the "coagulopathy group" stratified according to peri-interventional substitution policy are shown in $>$ Table 3 . There was no difference in incidence of bleeding and risk for mortality between EVL procedures with and without substitution of coagulation products ( $>$ Table 3 ).

\section{Discussion}

The results of this retrospective two-center study suggest that severity of liver disease (as indicated by serum bilirubin levels) and size of esophageal varices, rather than changes in coagulation test results, are associated with UGIB after EVL in patients with liver cirrhosis.

EVL is considered standard of care in cirrhosis patients with medium to large varices to reduce bleeding incidence from esophageal varices. Although this procedure is relatively safe, the literature reports periprocedural bleeding rates exceeding $6 \%$ [9]. As changes in coagulation test results are common in advanced liver disease $[12,21]$, it can be hypothesized that EVL-related bleeding in cirrhosis patients may, at least partly, be attributable to these coagulation disturbances.

However, our data do not support this hypothesis. Although INR was significantly associated with bleeding complications in the univariate analysis, the absolute difference in INR between 
patients who did and did not develop bleeding after EVL was clinically negligible ( $\triangleright$ Table 2 ). Moreover, this association did not remain significant after multivariate adjustment. Similarly, there was no significant association between platelet counts and bleeding events ( $>$ Table 2 ). We did observe lower fibrinogen levels in patients with UGIB following EVL ( Table 1); however, no statistically significant association between fibrinogen levels and occurrence of early UGIB after EVL was observed $(\triangleright$ Table 2). Serum bilirubin levels prior to EVL and presence of varices grade III/IV were identified as independent factors significantly associated with UGIB after EVL in stable cirrhosis patients ( $>$ Table 2 ).

The finding that standard coagulation tests are a poor indicator of bleeding complications is consistent with the literature $[22,23]$. A study in 150 patients suggested that post-EVL ulcer bleeding was associated with Child $C$ status but not with standard coagulation tests [24]. As a consequence, the use of INR in particular for prediction of bleeding in cirrhosis patients is no longer recommended $[5,25]$. In patients with stable liver cirrhosis, the concept of rebalanced hemostasis is increasingly acknowledged, which suggests that both pro- and anticoagulatory factors are decreased, resulting in a balanced coagulation despite abnormal routine coagulation tests $[12,15]$. However, in clinical practice, substitution of coagulation products (especially before invasive procedures) is often triggered by changes in routine coagulation test results (e.g. platelet count $<50 \times$ $10^{9} / \mathrm{L}$, INR $>1.5$, or fibrinogen $<1 \mathrm{~g} / \mathrm{L}$, respectively) in patients with liver cirrhosis, and is still suggested by some guidelines/ consensus statements [26,27].

Yet, evidence supporting the prophylactic substitution of coagulation products to prevent bleeding events in cirrhosis is lacking [28]. The infusion of platelets and/or FFP has only marginal effects on platelet count and INR, respectively $[22,29]$. In a randomized, controlled trial, the use of thrombopoietin receptor agonist eltrombopag prior to planned/elective, invasive procedures in patients with cirrhosis and thrombocytopenia had no effect on bleeding events (despite a significant increase in platelet count), but was associated with an increased risk for venous thrombosis [30]. Moreover, volume load associated with blood product substitution, especially FFPs, may paradoxically trigger bleeding via an increase in portal pressure [31]. These data clearly call into question the liberal substitution regimens frequently applied to cirrhosis patients with abnormal standard coagulation tests. Recent studies advocate viscoelastic testing as an appropriate alternative method to evaluate bleeding risk in patients with cirrhosis $[32,33]$. It has been shown that substitution guided by viscoelastic testing reduces transfusions of blood/coagulation products without increasing the risk for bleeding [34,35]. However, these studies do not answer the question of whether preinterventional prophylactic substitution of coagulation products reduces the risk of bleeding.

Thus, as a secondary aim, our study evaluated the peri-interventional use of coagulation products, and its potential effect on bleeding complications after EVL. For this purpose, we defined a "coagulopathy group" by INR > 1.5 and/or platelet count $<50 \times 10^{9} / \mathrm{L}$, which are common threshold values for substitu- tion of coagulation products prior to invasive procedures. In this group, substitution of blood/coagulation products was not associated with fewer bleeding events following EVL, despite comparable baseline characteristics (except for a small but significant difference in platelet count). Similarly, there was no difference in procedure-associated mortality between patients who received substitution of coagulation products and those who did not ( $>$ Table 3 ). Thus, our data do not support the prophylactic use of FFPs and/or platelets before prophylactic EVL in cirrhosis patients. However, future prospective studies are needed to evaluate potential risks and benefits of prophylactic substitution strategies in patients with liver cirrhosis.

Importantly, only a few bleeding events occurred within 24 hours after EVL. Thus, given the limited half-life of substituted coagulation products, the rationale of preinterventional blood product substitution is even more questionable.

Interestingly, larger size of esophageal varices was associated with a higher incidence of procedure-associated bleeding events. Considering that variceal size may be associated with portal pressure [36], this finding supports the established concept that the degree of portal hypertension is predictive of bleeding risk [37]. Conversely, we did not observe an association between platelet count and UGIB after EVL in our patients. This is particularly interesting as platelet counts are considered inversely associated with portal pressure [38], and have been linked to bleeding after invasive procedures [39]. Yet, all of our EVL patients had signs of portal hypertension (such as varices) and platelet counts were generally low. Thus, the preselection of our patients may have limited the range of platelet counts evaluated in our study. Nevertheless, in our portal-hypertensive patient sample of interest, platelet counts were not associated with bleeding risk.

The benefit of PPI therapy in cirrhosis patients remains a matter of debate. In a small study, pantoprazole therapy after prophylactic EVL was significantly associated with smaller post-banding ulcers but had no effect on bleeding complications [11]. A retrospective cohort study suggested that patients without PPI had an increased risk for post-EVL bleeding [40], which was also suggested by a recent meta-analysis [41]. However, in this meta-analysis, the authors found no beneficial effects of PPI in preventing death, ulcers or pain following EVL [41]. In our study, we did not observe an association between periprocedural PPI use and bleeding complications in cirrhosis patients undergoing EVL ( $\triangleright$ Table 2 ). Almost $80 \%$ of our patients received PPI therapy. Given the potentially harmful effects [42], PPI use in cirrhosis patients - not only following EVL requires further investigation.

Some limitations need to be considered when interpreting the results of this study. First, despite the relatively large size of the sample, the study was not sufficiently powered to draw definite conclusions and recommendations for clinical practice owing to the low bleeding rate following EVL. However, our results are based on a large patient sample and add to the increasing number of studies challenging the role of standard coagulation tests and routine blood product substitution in cirrhosis, and are a good basis for further analyses in this field. Second, the retrospective design implies inherent limitations 
and potential for bias. However, our study comprises a large number of patients and procedures from two academic medical centers. All data were documented in the patient data management systems in a prospective manner by trained staff, but were extracted later for this analysis. Third, the use of coagulation/blood products and PPIs was not standardized across patients and medical centers. Nonetheless, our data warrant the evaluation of a real-life substitution policy, which is frequently based on changes in routine coagulation test results, especially platelets and INR. Fourth, assessment/measurement of portal hypertension was not routinely performed in our patients. Fifth, residual confounding is always a matter of concern and cannot be entirely excluded. Finally, post-procedural dietary management may affect rebleeding as suggested by a recent case report [43]. However, adherence to dietary recommendations was not assessed in our study.

In conclusion, our data show that EVL is generally a safe procedure in patients with liver cirrhosis. Bleeding complications after EVL, observed in approximately $5 \%$ of procedures, were mainly determined by severity of liver disease and variceal size. Moreover, in a predefined "coagulopathy group" (INR > 1.5 and/ or platelets $<50 \times 10^{9} / \mathrm{L}$ ), we did not observe a difference in the incidence of UGIB after EVL between patients who received blood/coagulation products and those who did not. Thus, our study raises further doubts about the rationale for "prophylactic" blood/coagulation product substitution guided by standard coagulation tests in patients with liver cirrhosis undergoing planned/elective procedures.

\section{Competing interests}

The authors declare that they have no conflicts of interest.

\section{References}

[1] Simonetto DA, Shah VH, Kamath PS. Primary prophylaxis of variceal bleeding. Clin Liver Dis 2014; 18: 335-345

[2] Sharma M, Singh S, Desai V et al. Comparison of therapies for primary prevention of esophageal variceal bleeding: a systematic review and network meta-analysis. Hepatology 2019; 69: 1657-1675

[3] Garcia-Pagan JC, De Gottardi A, Bosch J. Review article: the modern management of portal hypertension - primary and secondary prophylaxis of variceal bleeding in cirrhotic patients. Aliment Pharmacol Ther 2008; 28: 178-186

[4] D’Amico G, Luca A. Natural history. Clinical-haemodynamic correlations. Prediction of the risk of bleeding. Baillieres Clin Gastroenterol 1997; 11: 243-256

[5] de Franchis R, Baveno VIF. Expanding consensus in portal hypertension: report of the Baveno VI Consensus Workshop: Stratifying risk and individualizing care for portal hypertension. J Hepatol 2015; 63 : 743-752

[6] de Franchis R, Pascal JP, Ancona E et al. Definitions, methodology and therapeutic strategies in portal hypertension. A consensus development workshop, Baveno, Lake Maggiore, Italy, April 5 and 6, 1990. J Hepatol 1992; 15: 256-261
[7] Laine L, el-Newihi HM, Migikovsky B et al. Endoscopic ligation compared with sclerotherapy for the treatment of bleeding esophageal varices. Ann Intern Med 1993; 119: 1-7

[8] Baroncini D, Milandri GL, Borioni D et al. A prospective randomized trial of sclerotherapy versus ligation in the elective treatment of bleeding esophageal varices. Endoscopy 1997; 29: 235-240

[9] Sarin SK, Govil A, Jain AK et al. Prospective randomized trial of endoscopic sclerotherapy versus variceal band ligation for esophageal varices: influence on gastropathy, gastric varices and variceal recurrence. J Hepatol 1997; 26: 826-832

[10] Stiegmann GV, Goff JS, Michaletz-Onody PA et al. Endoscopic sclerotherapy as compared with endoscopic ligation for bleeding esophageal varices. N Engl J Med 1992; 326: 1527-1532

[11] Shaheen NJ, Stuart E, Schmitz SM et al. Pantoprazole reduces the size of postbanding ulcers after variceal band ligation: a randomized, controlled trial. Hepatology 2005; 41: 588-594

[12] Caldwell SH, Hoffman M, Lisman T et al. Coagulation disorders and hemostasis in liver disease: pathophysiology and critical assessment of current management. Hepatology 2006; 44: 1039-1046

[13] Afdhal N, McHutchison J, Brown R et al. Thrombocytopenia associated with chronic liver disease. J Hepatol 2008; 48: 1000-1007

[14] Drolz A, Horvatits T, Roedl K et al. Coagulation parameters and major bleeding in critically ill patients with cirrhosis. Hepatology 2016; 64: 556-568

[15] Lisman T, Caldwell SH, Burroughs AK et al. Hemostasis and thrombosis in patients with liver disease: the ups and downs. J Hepatol 2010; 53: $362-371$

[16] Kamath PS, Wiesner RH, Malinchoc M et al. A model to predict survival in patients with end-stage liver disease. Hepatology 2001; 33: 464-470

[17] Paquet KJ. Prophylactic endoscopic sclerosing treatment of the esophageal wall in varices - a prospective controlled randomized trial. Endoscopy 1982; 14: 4-5

[18] Sey MSL, Mohammed SB, Brahmania M et al. Comparative outcomes in patients with ulcer- vs non-ulcer-related acute upper gastrointestinal bleeding in the United Kingdom: a nationwide cohort of 4474 patients. Aliment Pharmacol Ther 2019; 49: 537-545

[19] Ripatti S, Palmgren J. Estimation of multivariate frailty models using penalized partial likelihood. Biometrics 2000; 56: 1016-1022

[20] Austin PC, Wagner P, Merlo ]. The median hazard ratio: a useful measure of variance and general contextual effects in multilevel survival analysis. Stat Med 2017; 36: 928-938

[21] Tripodi A. Tests of coagulation in liver disease. Clin Liver Dis 2009; 13: 55-61

[22] Napolitano G, lacobellis A, Merla A et al. Bleeding after invasive procedures is rare and unpredicted by platelet counts in cirrhotic patients with thrombocytopenia. Eur J Intern Med 2017; 38: 79-82

[23] De Gottardi A, Thevenot T, Spahr L et al. Risk of complications after abdominal paracentesis in cirrhotic patients: a prospective study. Clin Gastroenterol Hepatol 2009; 7: 906-909

[24] Vieira da Rocha EC, D’Amico EA, Caldwell SH et al. A prospective study of conventional and expanded coagulation indices in predicting ulcer bleeding after variceal band ligation. Clin Gastroenterol Hepatol 2009; 7: 988-993

[25] Garcia-Tsao G, Abraldes JG, Berzigotti A et al. Portal hypertensive bleeding in cirrhosis: risk stratification, diagnosis, and management: 2016 practice guidance by the American Association for the study of liver diseases. Hepatology 2017; 65: 310-335

[26] Tripathi D, Stanley AJ, Hayes PC et al. U.K. guidelines on the management of variceal haemorrhage in cirrhotic patients. Gut 2015; 64: 1680-1704 
[27] Götz M, Anders M, Biecker E et al. [S2k Guideline Gastrointestinal Bleeding - Guideline of the German Society of Gastroenterology DGVS]. Z Gastroenterol 2017; 55: 883-936

[28] Stanworth SJ, Grant-Casey J, Lowe D et al. The use of fresh-frozen plasma in England: high levels of inappropriate use in adults and children. Transfusion 2011; 51: 62-70

[29] Abdel-Wahab OI, Healy B, Dzik WH. Effect of fresh-frozen plasma transfusion on prothrombin time and bleeding in patients with mild coagulation abnormalities. Transfusion 2006; 46: 1279-1285

[30] Afdhal NH, Giannini EG, Tayyab G et al. Eltrombopag before procedures in patients with cirrhosis and thrombocytopenia. $N$ Engl J Med 2012; 367: 716-724

[31] Giannini EG, Stravitz RT, Caldwell SH. Correction of hemostatic abnormalities and portal pressure variations in patients with cirrhosis. Hepatology 2014; 60: 1442

[32] Bedreli S, Sowa JP, Malek S et al. Rotational thromboelastometry can detect factor XIII deficiency and bleeding diathesis in patients with cirrhosis. Liver Int 2017; 37: 562-568

[33] Tripodi A, Primignani M, Chantarangkul V et al. The coagulopathy of cirrhosis assessed by thromboelastometry and its correlation with conventional coagulation parameters. Thromb Res 2009; 124: 132136

[34] De Pietri L, Bianchini M, Montalti R et al. Thrombelastography-guided blood product use before invasive procedures in cirrhosis with severe coagulopathy: a randomized, controlled trial. Hepatology 2016; 63: 566-573

[35] Saner FH, Bezinover D, Sakai T. Assessment of haemostasis in patients with liver cirrhosis. Eur J Anaesthesiol 2017; 34: 33-34
[36] Wadhawan M, Dubey S, Sharma BC et al. Hepatic venous pressure gradient in cirrhosis: correlation with the size of varices, bleeding, ascites, and child's status. Dig Dis Sci 2006; 51: 2264-2269

[37] Garcia-Tsao G, Groszmann RJ, Fisher RL et al. Portal pressure, presence of gastroesophageal varices and variceal bleeding. Hepatology 1985; 5: 419-424

[38] Berzigotti A, Seijo S, Arena U et al. Elastography, spleen size, and platelet count identify portal hypertension in patients with compensated cirrhosis. Gastroenterology 2013; 144: 102-111. e101

[39] Li J, Han B, Li H et al. Association of coagulopathy with the risk of bleeding after invasive procedures in liver cirrhosis. Saudi J Gastroenterol 2018; 24: 220-227

[40] Kang SH, Yim H], Kim SY et al. Proton pump inhibitor therapy is associated with reduction of early bleeding risk after prophylactic endoscopic variceal band ligation: a retrospective cohort study. Medicine (Baltimore) 2016; 95: e2903

[41] Zhu J, Qi X, Yu H et al. Acid suppression in patients treated with endoscopic therapy for the management of gastroesophageal varices: a systematic review and meta-analysis. Expert Rev Gastroenterol Hepatol 2018; 12: 617-624

[42] Dultz G, Piiper A, Zeuzem S et al. Proton pump inhibitor treatment is associated with the severity of liver disease and increased mortality in patients with cirrhosis. Aliment Pharmacol Ther 2015; 41: 459-466

[43] Li Y, Guo X, Bai Z et al. Banana may be forbidden after endoscopic variceal ligation: a case report. Transl Gastroenterol Hepatol 2019; 4: 13 